\title{
Parabrachial Innervation of the Cat's Dorsal Lateral Geniculate Nucleus: An Electron Microscopic Study Using the Tracer Phaseolus vulgaris Leucoagglutinin (PHA-L)
}

\author{
Josephine B. Cucchiaro, Daniel J. Uhlrich, and S. Murray Sherman \\ Department of Neurobiology and Behavior, State University of New York at Stony Brook, Stony Brook, New York 11794- \\ 5230
}

Ascending pathways from the brain stem play a key role, generally facilitatory, in controlling the transmission of retinal information through the lateral geniculate nucleus to the visual cortex (for reviews, see Singer, 1977; Burke and Cole, 1978; Sherman and Koch, 1986). In order to characterize the morphological basis of this brain-stem control, we used the electron microscope to study synaptic terminals labeled anterogradely from injections of the tracer Phaseolus vulgaris leucoagglutinin into the parabrachial region of the brain stem. The labeled axons, which are fine and unmyelinated in our material, form conventional synaptic contacts onto both relay cells and interneurons. These connections are surprisingly selective for certain postsynaptic elements such as the dendritic shafts and appendages of relay cells and the presynaptic dendritic terminals of interneurons. That is, the morphology of contacts made from parabrachial axons varies with the specific postsynaptic profile. Even a single axon can form symmetrical contacts onto F2 terminals, which are synaptic terminals deriving from dendrites of interneurons, and dendritic shafts of relay $X$ cells, and form asymmetrical contacts onto dendritic appendages of the same relay $X$ cells. Reconstructions of the dendritic segments postsynaptic to the labeled terminals show that the dendritic appendages receive retinal and parabrachial input in triadic relationships with $\mathbf{F 2}$ terminals: a retinal or parabrachial axon contacts the F2 terminal, and the F2 terminal plus the retinal or parabrachial axon contact the dendritic appendage. This positioning of the parabrachial innervation is well suited for control of retinal transmission. Finally, the dual morphology of the parabrachial synaptic contacts suggests that their actions may differ depending on the postsynaptic target.

Neurons located in the parabrachial region and locus coeruleus innervate all known nuclei of the thalamus that are engaged in visual processing, including all laminae of the cat's dorsal lateral geniculate nucleus, and lateral posterior-pulvinar complex (Leger et al., 1975; Ahlsen and Lo, 1982; Hughes and Mullikin, er et al., 1975; Ahlsen and Lo, 1982; Hughes and Mullikin, 1984; De Lima and Singer, 1987; Smith et al., 1988; Uhlrich et al., 1988). Activation of neurons in these regions of the cat's

\footnotetext{
Received Nov. 9, 1987; revised May 4, 1988; accepted May 10, 1988.

This work was supported by USPIIS Grants EY03604, EY06610, and EY05754. We thank Sue Van Horn, Joan Sommermeyer, and Rachel Skovira for their excellent technical assistance.

Correspondence should be addressed to Dr. J. B. Cucchiaro at the above address. Copyright (c) 1988 Society for Neuroscience $0270-6474 / 88 / 124576-13 \$ 02.00 / 0$
}

brain stem generally facilitates the transmission of retinal signals through the cat's lateral geniculate nucleus to the visual cortex (for reviews, see Singer, 1977; Burke and Cole, 1978; Sherman and Koch, 1986). The majority of these brain-stem neurons that innervate the A-laminae of the lateral geniculate nucleus are cholinergic and are located in the parabrachial region, although some noradrenergic neurons in the parabrachial rcgion and locus coeruleus also innervate these geniculate laminae (De Lima and Singer, 1987).

Some investigators claim that the brain-stem facilitatory effects on geniculate relay neurons are accomplished indirectly by disinhibition (i.e., by directly inhibiting local inhibitory GABAergic neurons) without direct brain-stem input to the relay cells (Ahlsen and Lo, 1982; Ahlsen et al., 1984). Others have shown evidence for a direct facilitation of relay neurons by brain-stem axons (Sillito et al., 1983; De Lima et al., 1985; Eysel et al., 1986; McCormick and Prince, 1987). To address this controversy and to begin characterizing the morphological basis for the control by these brain-stem neurons of geniculate relay cells, we used the electron microscope to study synaptic terminals labeled anterogradely from injections of the tracer Phaseolus vulgaris leucoagglutinin (PHA-L) into the parabrachial region. This represents an extension of the light microscopic observations reported in the preceding paper (Uhlrich et al., 1988). A preliminary report of our electron microscopic observations has appeared (Cucchiaro et al., 1986).

\section{Materials and Methods}

PHA-L injection to the parabrachial region. In one cat, PHA-L was iontophoresed into a single site at the dorsal border of the brachium conjunctivum, $4 \mathrm{~mm}$ lateral to the midline. We used our previously described parameters for this injection (Uhlrich et al., 1988). After a 7 d survival period, the cat was sacrificed for neurohistological processing. We used an immunohistochemical protocol similar to that described previously (Gerfen and Sawchenko, 1984; Uhlrich et al., 1988) with modifications for electron microscopy (Eldred et al., 1983).

Only the modifications are described here. The animal was perfused with $4 \%$ paraformaldehyde and $0.05 \%$ glutaraldehyde (EM Grade) in sodium phosphate buffer at $\mathrm{pH} 7.4$, and the brain was stored overnight at $4^{\circ} \mathrm{C}$ in $4 \%$ paraformaldehyde buffered with $0.05 \mathrm{M}$ sodium borate at $\mathrm{pH} 9.5$. On the following day, we used a Vibratome to cut $50 \mu \mathrm{m}$ coronal sections through the lateral geniculate nucleus ipsilateral to the PHA-L injection site. We exposed the sections to a graded series of alcohols $(10,20,40,20$, and $10 \%)$ for $5 \mathrm{~min}$ each, and we avoided all use of Triton $X-100$. The sections were incubated in primary antibodies directed against PHA-L (Vector Labs) for $72 \mathrm{hr}$. We then visualized the PHA-L immunohistochemically using the avidin-biotin method (Gerfen and Sawchenko, 1984; Uhlrich et al., 1988) and reaction of the attached HRP with diaminobenzidine.

The sections were examined under the light microscope to identify those with labeled fibers. These sections were postfixed with osmium 


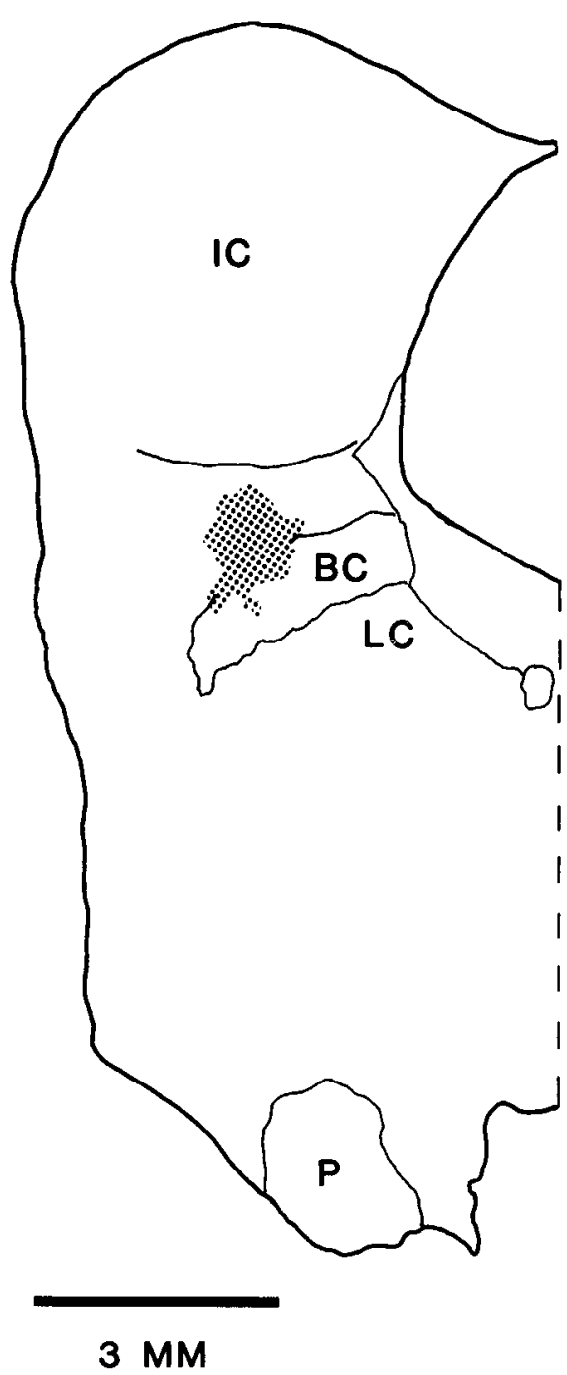

Figure 1. Line drawing of a section through the cat's midbrain showing the PHA-L injection site (stippled area). IC, inferior colliculus; $B C$, brachium conjunctivum; $L C$, locus coeruleus; $P$, pyramidal tract.

tetroxide, embedded in Epon, and prepared for electron microscopic analysis. Because our immunohistochemical protocol often limited penetration of the antibodies to 8-10 $\mu \mathrm{m}$ from the surface of each $50 \mu \mathrm{m}$ section, single axons from this animal could not be reconstructed through serial sections at the light microscopic level. Our electron microscopic analysis focused instead on limited extents of individual, labeled axons located near the surfaces of the thick sections.

Serial thin sections were cut and mounted onto Formvar-coated, slotted grids and stained with uranyl acetate and lead citrate. We examined and photographed every section. We analyzed 2 blocks of tissue, each laken from the binocular portion of the geniculate A-laminae; the blocks were separated from one another by about $2 \mathrm{~mm}$. This separation precludes the possibility that reconstructed postsynaptic dendrites from the 2 regions belong to a single geniculate cell. The first block contained 7 labeled axon segments that we traced as far as possible through a single progression of 290 serial sections. Likewise, we traced 5 labeled axon segments as far as we could through the second block in a single sequence of 144 serial sections. We reconstructed portions of 24 dendritic arbors that were postsynaptic to labeled terminals.

We determined the sizes of labeled terminals at sites of synaptic contact by measuring the long and the short diameters of each terminal on prints at a magnification of 27,000 ; we averaged these values to arrive at a single measure of diameter. We also assessed the extent of postsynaptic density for each contact that was cut perpendicular to the membranes and for which a complete series of sections through the synaptic contact site was available. The maximum extent of this density through the series was taken as the measure of its size. These measure-

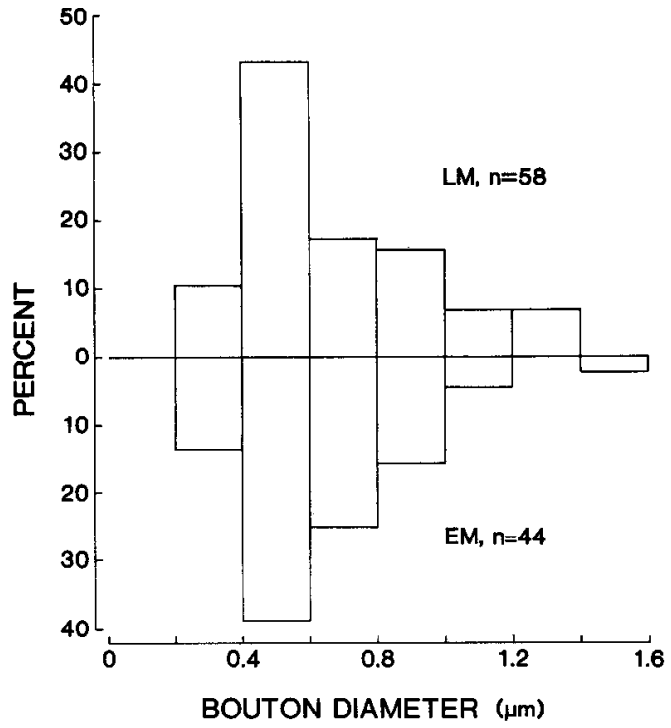

Figure 2. Diameter distributions of labeled parabrachial boutons measured with the light and electron microscopes ( $L M$ and $E M$, respectively).

ments were made from prints at a magnification of 46,000 . Two independent observers measured each synaptic contact. When the measurements by the 2 observers differed, the differences were almost always less than $5 \mathrm{~nm}$, and the average of the 2 measures was taken as our estimate of the extent of the postsynaptic density. From the same prints, we also estimated the widths of the synaptic clefts by calculating the widest distance between the pre- and postsynaptic membranes in each reconstructed synaptic contact zone.

PHA-L injections into the visual cortex. In a second cat, a small amount of PHA-L was injected into the visual cortex to label corticogeniculate axons and terminals in the lateral geniculate nucleus. The injection parameters and immunohistological processing from this cat were identical to those decribed above for the animal with PHA-L injected into the parabrachial region. Because the morphology of corticogeniculate terminals has been described from a variety of other techniques (Jones and Powell, 1969; Robson, 1983; Weber and Kalil, 1987), this material from the second cat served as a control against the possibility that PHA-L labeling itself alters the morphology of the synaptic contact zone.

Statistics. Unless otherwise noted, all statistical comparisons are based on the Mann-Whitney $U$ test.

\section{Results}

\section{General features of the PHA-L labeling}

Figure 1 shows the site of the injection of PHA-L in the parabrachial region. As described in the previous paper (Uhlrich et al., 1988), this injection labeled fibers in all thalamic nuclei involved in visual processing. The labeled parabrachial axons are fine and beaded, with en passant swellings $0.2-1.6 \mu \mathrm{m}$ in diameter. These swellings are the boutons seen with the light microscope, and synaptic contacts are often present at these boutons. In this single animal, we measured a random sample of labeled boutons with the light microscope and did the same for a second, smaller sample with the electron microscope. Figure 2 shows that the size distributions for the 2 populations are remarkably similar, suggesting that we have not overlooked a population of labeled boutons in our electron microscopic analysis.

\section{Identification of unlabeled synaptic terminals}

We used Guillery's (1969a) classification and terminology to identify synaptic terminals in the neuropil of the geniculate 

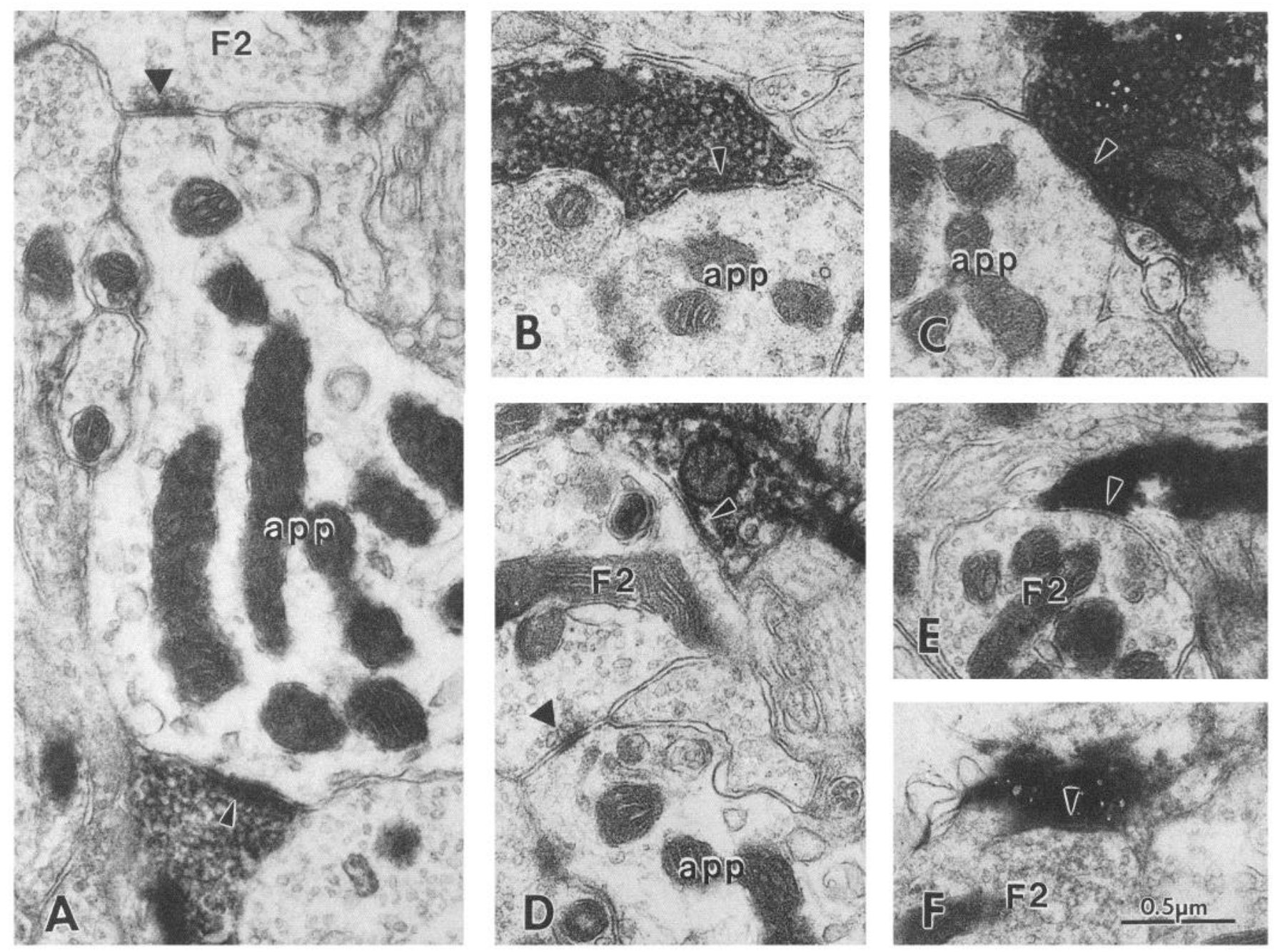

Figure 3. Electron micrographs of synaptic contacts from labeled parabrachial axons in the A-laminae of the cat's lateral geniculate nucleus. Synaptic contact sites are indicated by black triangles; these are outlined in white for the labeled terminals and are solid (i.e., not outlined) for other terminals. $A-C$, Contacts onto dendritic appendages (app) of presumed relay X cells. $D-F$, Contacts onto the presynaptic dendritic terminals $(F 2)$ of interneurons. Notice that the extent of the postsynaptic density varies with the postsynaptic partner. The labeled terminals in $A$ and $D$ are from a single axon reconstructed in Figure 4. This is an example of triadic parabrachial input: In $A$, the labeled terminal contacts the appendage of a relay cell (outlined triangle), and the same appendage also receives input from an F2 profile (solid triangle); in an adjacent section (D), the labeled axon contacts the same F2 terminal as in A (outlined triangle), which contacts the appendage (solid triangle). A retinal terminal (not illustrated) also enters into a triadic synaptic relationship with the same F2 profile and dendritic appendage shown in $A$ and $D$. Scale bar in $F$ also applies to $A-E$.

A-laminae. Both RLP (Round vesicles, Large profiles, Pale mitochondria) and RSD (Round vesicles, Small profiles, Dark mitochondria) teminals form asymmetrical synaptic contacts. Two types of F terminals (containing Flattened or pleomorphic vesicles), which form symmetrical synaptic contacts, are distinguished. F1 terminals have a darker cytoplasm and denser accumulation of vesicles than do $\mathrm{F} 2$ terminals. Also, F1 terminals are strictly presynaptic in relation to other profiles, while F2 terminals are both pre- and postsynaptic. RLP terminals are known to derive from the retina (Szentagothai et al., 1966; Guillery, 1969a, b; Robson and Mason, 1979) and F2 terminals are known to be the specialized dendritic appendages of geniculate interneurons (Famiglietti and Peters, 1972; Hamos et al., 1985; Montero, 1986). The sources of RSD and F1 terminals are less clear. Many RSD terminals are cortical in origin (Jones and Powell, 1969; Robson, 1983; Weber and Kalil, 1987), and most F1 terminals derive from local inhibitory neurons, in- cluding geniculate interneurons (Montero, 1987; J. E. Hamos, unpublished observations) and cells of the nearby perigeniculate nucleus (Cucchiaro et al., 1985). However, we present evidence below that some of the terminals that would be identified in unlabeled material as RSD or F1 terminals may have their origin in the parabrachial region of the brain stem.

\section{Synaptic circuitry of the labeled parabrachial axons}

Labeled synaptic terminals. We found that labeled terminals form synaptic contacts onto dendritic shafts and appendages of presumed geniculate relay cells and also onto $\mathrm{F} 2$ terminals of interneurons. Figure $3, A-C$ illustrates synaptic contacts from the labeled terminals onto dendritic appendages, and Figure 3, $D-F$ illustrates such contacts onto $F 2$ terminals. A series of consecutive sections through several synaptic contact zones are also shown in Figures 4 and 5. Synaptic vesicles are densely distributed throughout the labeled axons, filling both the swell- 
ings or boutons at which synaptic contacts are made, as well as the nonsynaptic areas between swellings (Fig. 6). The labeled axons have also been seen directly apposed to retinal terminals, but no synaptic specializations have been seen between these parabrachial axons and retinal terminals (Fig. 6B). The cytoplasm of each labeled bouton is completely filled with the dense, electron-opaque reaction product, leaving only mitochondria and synaptic vesicles devoid of label. Since features of the labeled terminals such as vesicle shape and cytoplasmic density could not be evaluated with any confidence, we instead focused on analysis of the cleft and postsynaptic portion of the synaptic specializations.

The axons labeled from the parabrachial region of the brain stem form synaptic contacts in the geniculate neuropil onto dendritic appendages, dendritic shafts, and F2 terminals. We found no synaptic contacts onto somata or any axonal profiles. Most of the synaptic contacts we have analyzed occur at boutons en passant along the length of the labeled axons, although one synaptic contact was made by a terminal appended to the main axon by a short stalk (Fig. 8). The synaptic contact from this appended terminal is illustrated in Figure $3 \mathrm{C}$. We have seen some swellings that do not appear to form synaptic contacts, while others can establish up to 3 synapses (Figs. 7-9).

Profiles postsynaptic to labeled terminals. Many synaptic contacts from the labeled parabrachial axons are formed onto dendritic appendages and shafts of geniculate neurons (Figs. 7-9). Our serial reconstructions show that labeled parabrachial axons are selective for particular postsynaptic targets that seem to be associated with distinctive geniculate cell types (Figs. 7-9). Dendrites postsynaptic to the labeled parabrachial input have clustered dendritic appendages and receive triadic retinal input. However, the dendritic segments illustrated are not from a single parent geniculate cell because the dendritic segments reconstructed in Figure 7 are about $2 \mathrm{~mm}$ away from the dendritic segments shown in Figures 8 and 9 (see Materials and Methods).

In some cases, we have been able to demonstrate that the dendritic shafts and appendages belong to the same postsynaptic neuron and that the appendages of these cells are clustered in complex arrays. Reconstructions of portions of these postsynaptic neurons indicate that they receive most of their retinal inputs onto their dendritic appendages in triadic relationships with F2 terminals (Famiglietti and Peters, 1972). That is, a retinal terminal contacts an F2 terminal, and both of these terminals contact the same dendritic appendage. In the reconstructions shown in Figures 7-9, 15 of the 18 retinal contacts shown onto the dendritic segments that are postsynaptic to the parabrachial input are involved in such triadic relationships. These triadic synapses usually occur within glomerular zones, which are regions of complex synaptic relationships (Szentagothai et al., 1966). Synapses from labeled parabrachial axons are often peripherally located in these same glomeruli. An example is shown in Figure 10.

The labeled terminals from parabrachial axons also synaptically contact F2 terminals. Because these F2 terminals are connected to each other and to their parent dendrites by long, thin processes (see Hamos et al., 1985; Montero, 1986), we were unable to reconstruct them further. Often the same labeled axon contacts an F2 terminal and a dendritic appendage, with a synaptic contact formed from the F2 terminal onto the dendritic appendage (Figs. 3, 7-9). This represents a triadic arrangement that is similar to the one described above for retinal axons, except that for retinal axons, the same terminal is presynaptic
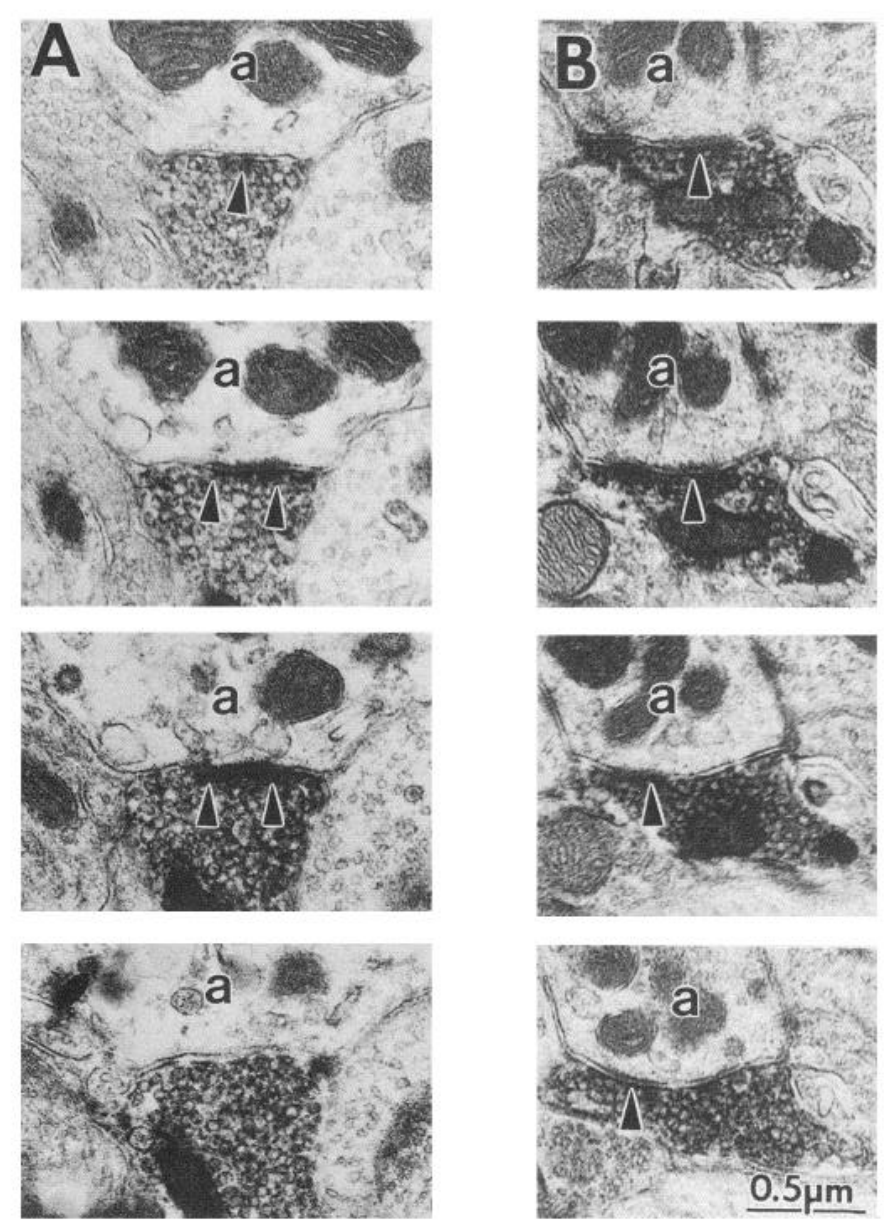

Figure 4. Electron micrographs of serial sections through 2 asymmetrical synapses formed by parabrachial axons onto dendritic appendages $(a)$. $A$, Four sections through the synaptic contact zone (arrowheads) of the synapse shown in Figure $3 A$. Synaptic vesicles densely fill the terminal. Because the postsynaptic density is comparatively thick in the second panel, this synapse has been classified as asymmetrical. However, the 2 adjacent sections have little density associated with the postsynaptic membrane, and any of these sections in isolation might lead to an inappropriate identification of this contact as symmetrical. The synaptic cleft of this contact is relatively wide. $B$, Four sections through a synaptic contact zone (arrowheads). The terminal is filled with synaptic vesicles, and dark mitochondria can be identified in the terminal when compared with the pale mitochondrion to the lower left. The synaptic cleft of this contact is relatively narrow compared with that shown in $A$, but there is a relatively thick postsynaptic density, leading to the contact's identification as asymmetrical. Scale bar in the bottom panel of $B$ applies to all panels.

to both the F2 terminal and the dendritic appendage; for parabrachial axons, different terminals are often presynaptic to each structure, although we have seen cases for which a single parabrachial terminal is presynaptic to both an F2 terminal and a dendritic appendage. Often a retinal terminal and parabrachial axon will synapse on the same F2 terminal and dendritic appendage. Such arrangements are illustrated by electron micrographs in Figure 3 (see the legend for details), by the reconstruction in Figure 8, and by the schematic drawing in Figure 11.

\section{Morphometry of synaptic contacts from parabrachial axons}

Postsynaptic densities. Synaptic contacts established from parabrachial axons onto dendritic appendages are striking because they usually exhibit a considerable postsynaptic density or thick- 
Figure 5. Electron micrographs of serial sections through 3 symmetrical synaptic contacts formed by parabrachial axons. $A$, Three sections through the contact zone of the synapse shown in Figure $3 D$, which is formed onto an F2 terminal $(F 2)$. This zone (arrowheads) is characterized by a narrow cleft between the pre- and postsynaptic membranes (identifiable in the $2 \mathrm{sec}$ tions indicated by arrowheads), by dense material in the cleft, and by a minimal postsynaptic density. Therefore, this contact is symmetrical. $B$ and $C$, Three adjacent sections through each of 2 synapses (arrowheads) formed onto dendritic shafts $(d)$ of geniculate cells in the extraglomerular neuropil. Both synapses have narrow clefts, with dense material in the cleft and minimal amounts of postsynaptic density. These have been classified as symmetrical synapses. Scale bar in the bottom panel of $C$ applies to all panels.
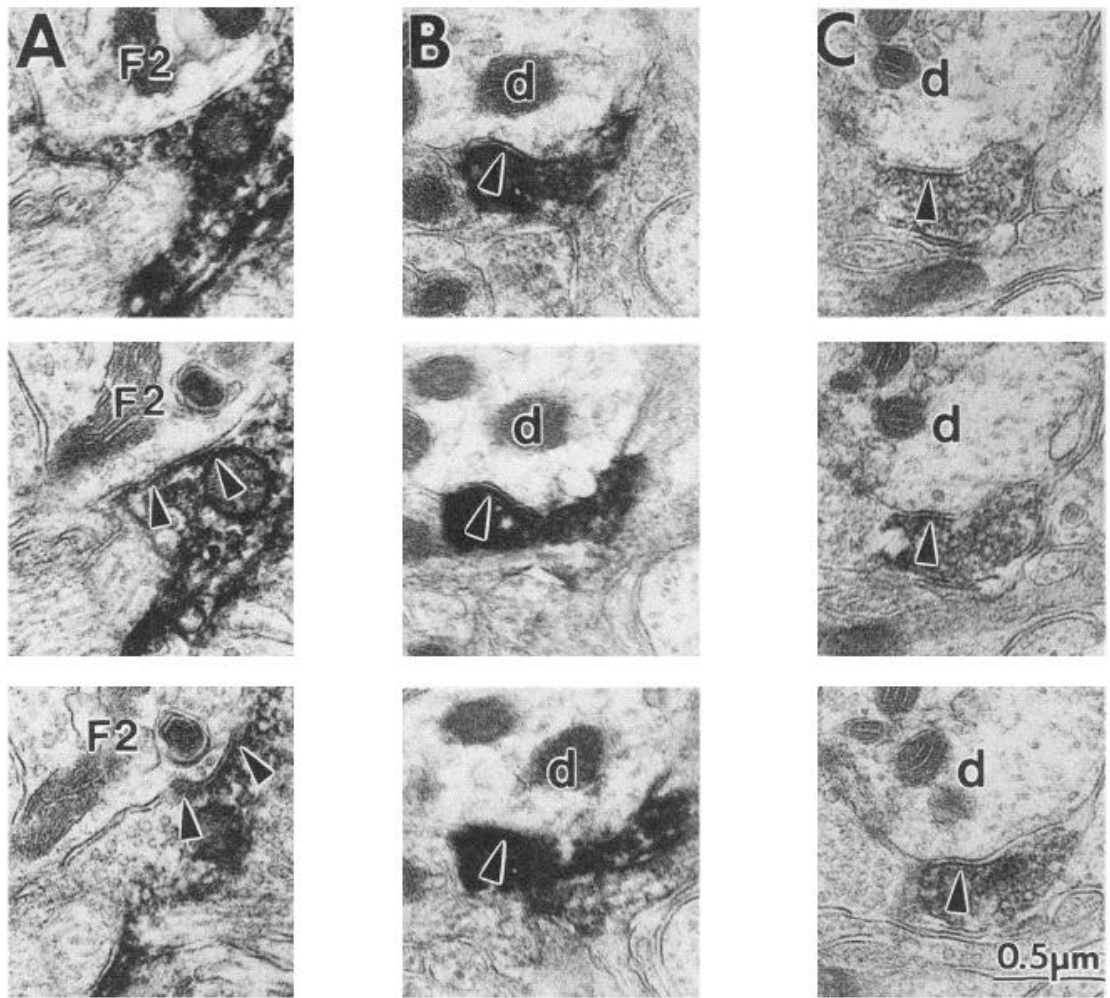

ening (Figs. 3, $A-C$; 4). In contrast, synaptic contacts formed onto F2 terminals and onto dendritic shafts are relatively small, being present in only 2 or 3 serial sections, and have little or no postsynaptic density associated with the contact zone (Figs. $3, D-F ; 5)$. Serial reconstructions show that a single labeled axon can contact all 3 classes of postsynaptic profile (i.e., dendritic shaft, dendritic appendage, and F2 terminal) and form contacts that include both thick and thin postsynaptic densities, the density thickness depending on the postsynaptic partner (Fig. 7). To verify this apparent distinction between thin and thick postsynaptic densities from the labeled terminal boutons, we quantitatively assessed the amount of postsynaptic density associated with synapses. This was done by measuring the postsynaptic thickening of synapses from unlabeled RSD, RLP, F2, and F1 terminals, as well as from terminals labeled with PHA-L from the visual cortex and from the parabrachial region (Fig. 12).

For the unlabeled terminals, the amount of postsynaptic thickening does indeed vary among the different terminal types. RSD terminals produce the thickest postsynaptic densities associated with synaptic contact sites, ranging from 30 to $65 \mathrm{~nm}$ (Fig. 12A), and F1 terminals have the thinnest densities, ranging from 15 to $30 \mathrm{~nm}$ (Fig. 12D). RLP and F2 terminals, both distinguishable by other morphological criteria, are intermediate in this regard (Fig. 12, B, C; see also Rapisardi and Lipsenthal, 1984). All of the pairwise comparisons of postsynaptic density among these terminal populations are statistically significant $(p<0.001$ for all comparisons except that between F1 and F2 terminals, for which $p<0.05$ ).

The terminals labeled from visual cortex have thick postsynaptic densities (Fig. 12E), very much in agreement with measurements for the unlabeled RSD terminals. Interestingly, although the mean postsynaptic density from unlabeled RSD terminals is not statistically different from those of labeled cortical terminals $(p>0.1)$, much more variance exists for the former population than for the latter ( $p<0.001$ on an $F$ test). This suggests the possibility that unlabeled RSD terminals are a more heterogeneous population than are identified cortical terminals, perhaps because these RSD terminals include some individuals from noncortical sources (see Discussion). Finally, the average postsynaptic density of the labeled cortical terminals is greater than that for each of the RLP, F2, and F1 terminal populations ( $p<0.001$ for each comparison).

On average, the labeled parabrachial terminals form contacts with thinner postsynaptic densities than do RSD, RLP, or labeled cortical terminals ( $p<0.001$ for each comparison), but no significant difference exists between the mean densities of parabrachial terminals and those of F2 and F1 terminals ( $p>$ 0.1 for each comparison). However, the labeled parabrachial terminals form synapses with a wide range of postsynaptic thicknesses (Fig. 12F). In fact, more variance is seen for this population than any other illustrated in Figure $12(p<0.01$ to $p<$ 0.001 on an $F$ test for each pairwise comparison). The differences in average postsynaptic density thickness and variance between labeled parabrachial and cortical terminals demonstrate that the labeling per se does not dictate the extent of postsynaptic thickening.

Finally, the thickness of the postsynaptic density for the labeled parabrachial terminals depends upon the postsynaptic partner (Fig. 12F). That is, the contacts onto dendritic appendages tend to display more postsynaptic thickening $(35-70 \mathrm{~nm})$ than do either those onto F2 terminals or those onto dendritic shafts $(15-35 \mathrm{~nm}$, with one contact of $53 \mathrm{~nm})$. These differences are statistically significant ( $p<0.001$ for each of the 2 comparisons). No density difference is seen between the synapses formed onto F2 terminals and those formed onto dendritic shafts 

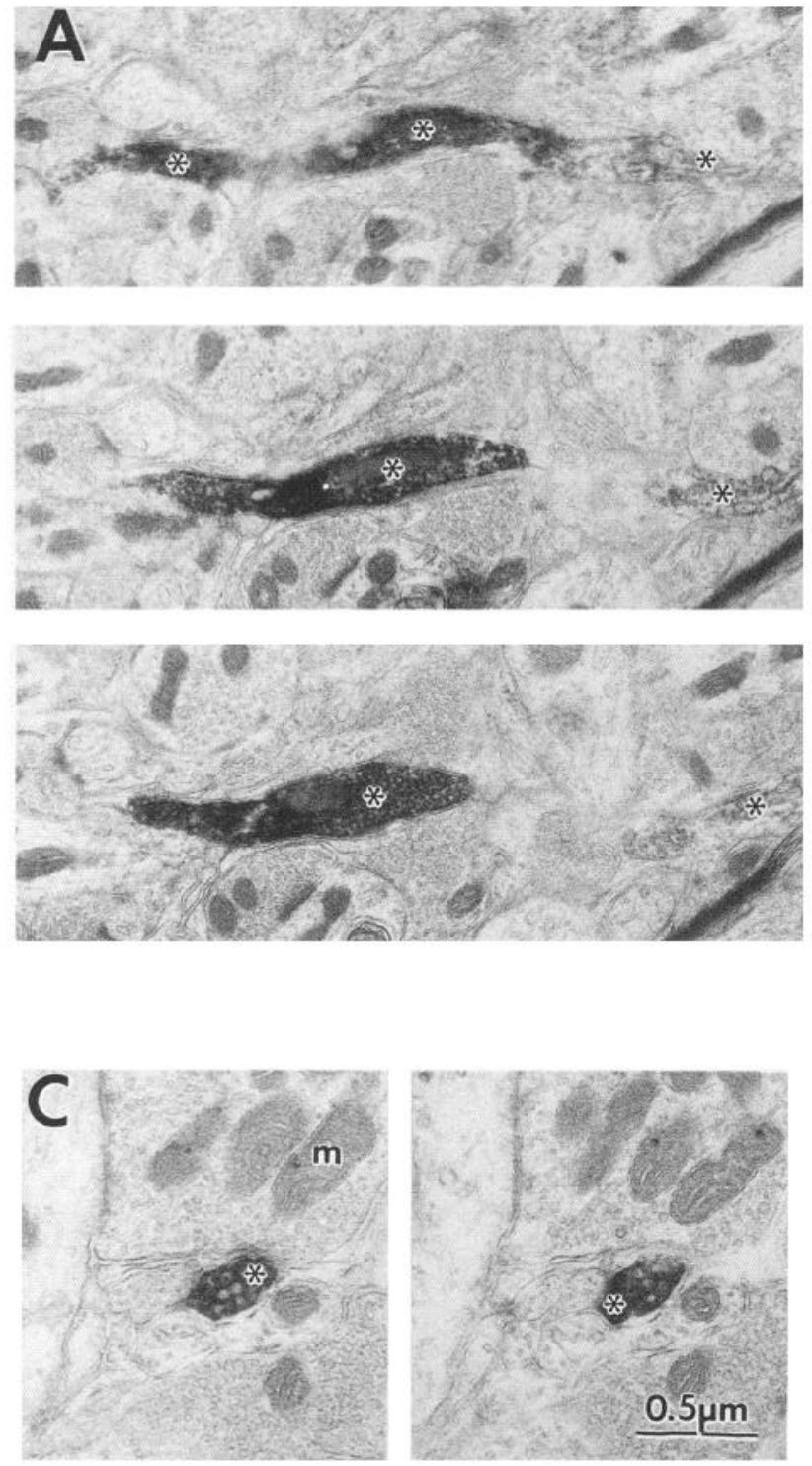

Figure 6. Electron micrographs of serial sections through 3 nonsynaptic regions of parabrachial axons. A, Longitudinal sections through part of an axon (asterisks) running through the extraglomerular neuropil. The widened portion of the axon is densely packed with vesicles and contains mitochondria, but it does not make any identifiable synapses. However, we cannot rule out the possibility that a synapse is present that has been cut tangentially. $B$, Three adjacent sections through part of a parabrachial axon (asterisks) entering a synaptic glomerulus. This axon is filled with vesicles and is directly apposed to a retinal terminal $(R L P)$, but the parabrachial axon does not form a synaptic contact with the retinal terminal. The latter terminal contacts (arrow) an F2 terminal $(F 2)$, which contacts (open triangle) a dendritic appendage (a). $C$, Two adjacent sections through a constricted portion of a labeled axon (asterisks). Synaptic vesicles are present in the axon even though it is barely wide enough to accommodate a single mitochondrion $(m)$, as seen in nearby profiles. Scale bar in the bottom panel of $B$ applies to all panels of $A$ and $B$; the scale bar in the right panel of $C$ applies to the left panel.

$(p>0.1)$. If these latter 2 groups are pooled, then they together form synapses with postsynaptic densities that are thinner than those formed from RSD terminals $(p<0.001)$ but equivalent to those formed by F1 terminals $(p>0.1)$. Conversely, the parabrachial synapses formed onto dendritic appendages exhibit postsynaptic densities that are equivalent to those formed from RSD terminals $(p>0.1)$ but thicker than those formed from F1 terminals $(p<0.001)$.

Width of synaptic cleft. We also measured the widths of the clefts associated with synaptic contacts for the various populations of labeled and unlabeled terminals. As can be seen in Figure 13, we found no correlation between cleft width and postsynaptic density for each of the 6 terminal populations ( $p$
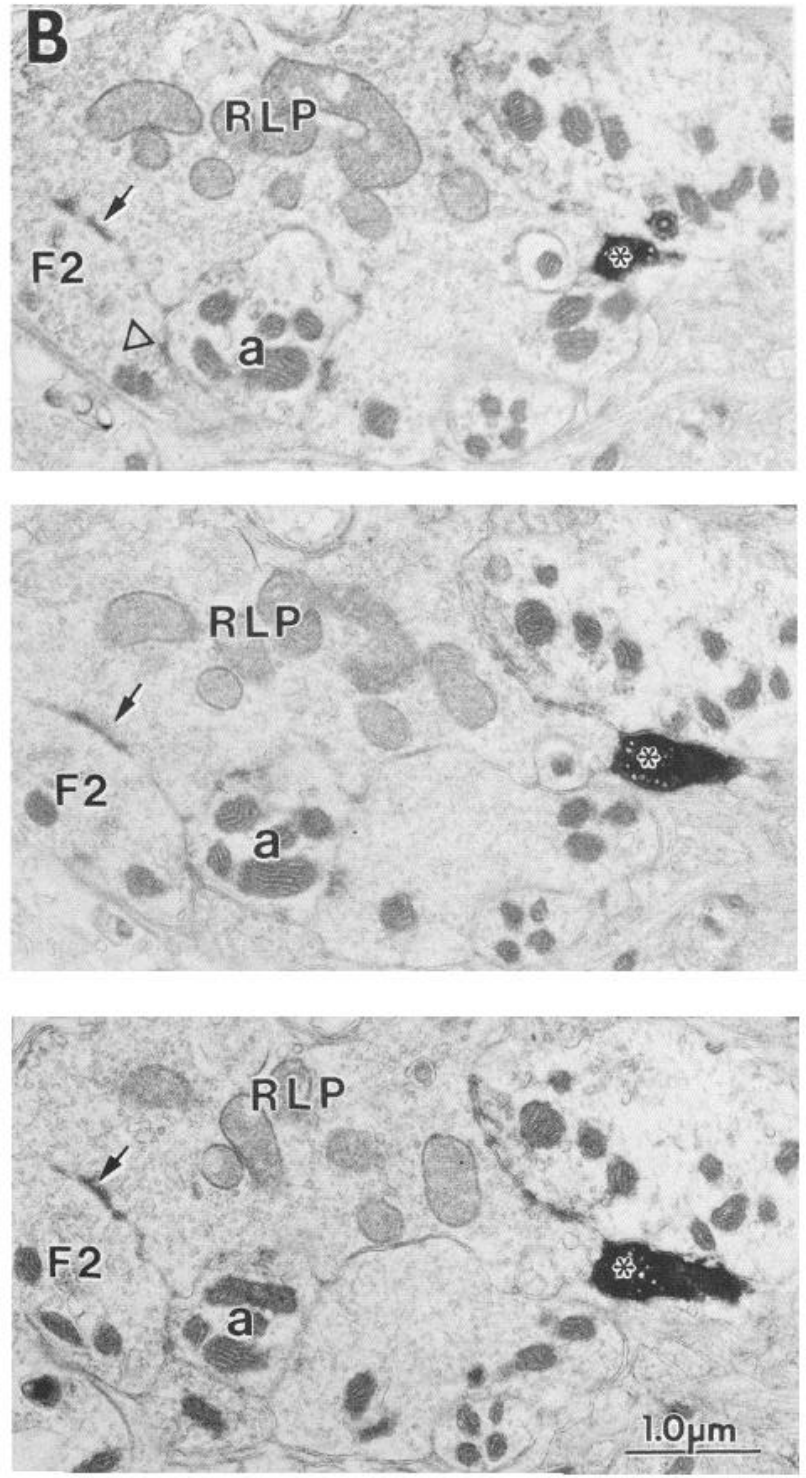

$>0.1$ for each correlation coefficient), an observation that supports a previous study of geniculate synapses (Rapisardi and Lipsenthal, 1984). Even when data from all of these synapses were pooled, no significant correlation emerged $(p>0.1)$.

We found that RSD terminals, which have the thickest postsynaptic densities, tend to have wider synaptic clefts than do F1 terminals, which have the thinnest postsynaptic densities ( $p$ $<0.01)$. The labeled cortical terminals may also have wider clefts than do F1 terminals $(p<0.05)$. However, on all other pairwise comparisons among the unlabeled terminal populations, and between the labeled cortical and RSD, RLP, or F2 terminals, no difference was evident in the mean width of their synaptic clefts ( $p>0.1$ on each comparison). 
Figure 7. Serial reconstructions of a labeled parabrachial axon (stippled overlay) and dendritic segments postsynaptic to it; the labeled axon is also reconstructed separately to the right. All synaptic contacts onto the dendritic segments are shown, including those from labeled parabrachial terminals (open stars in solid circles), from RLP terminals (solid squares), from F1 and F2 profiles (solid triangles), and from RSD terminals (solid circles). Also shown are triadic relationships involving retinal and parabrachial terminals with $\mathrm{F} 2$ terminals and the postsynaptic dendrites (overlapping symbols). Note that there are 3 dendritic segments that are disconnected due to incomplete reconstruction; however, each of the 3 receives triadic retinal input and innervation from a single parabrachial axon. The reconstruction at right shows all of the parabrachial axon's synaptic outputs. This parabrachial axon contacts dendritic appendages and shafts (open stars) of presumed relay cells as well as F2 terminals (open triangles) of intcrneurons. The asterisks indicate the synapses shown in Figure 3, $A$ and $D$, and the arrow points to the synapse shown in Figure $3 E$.

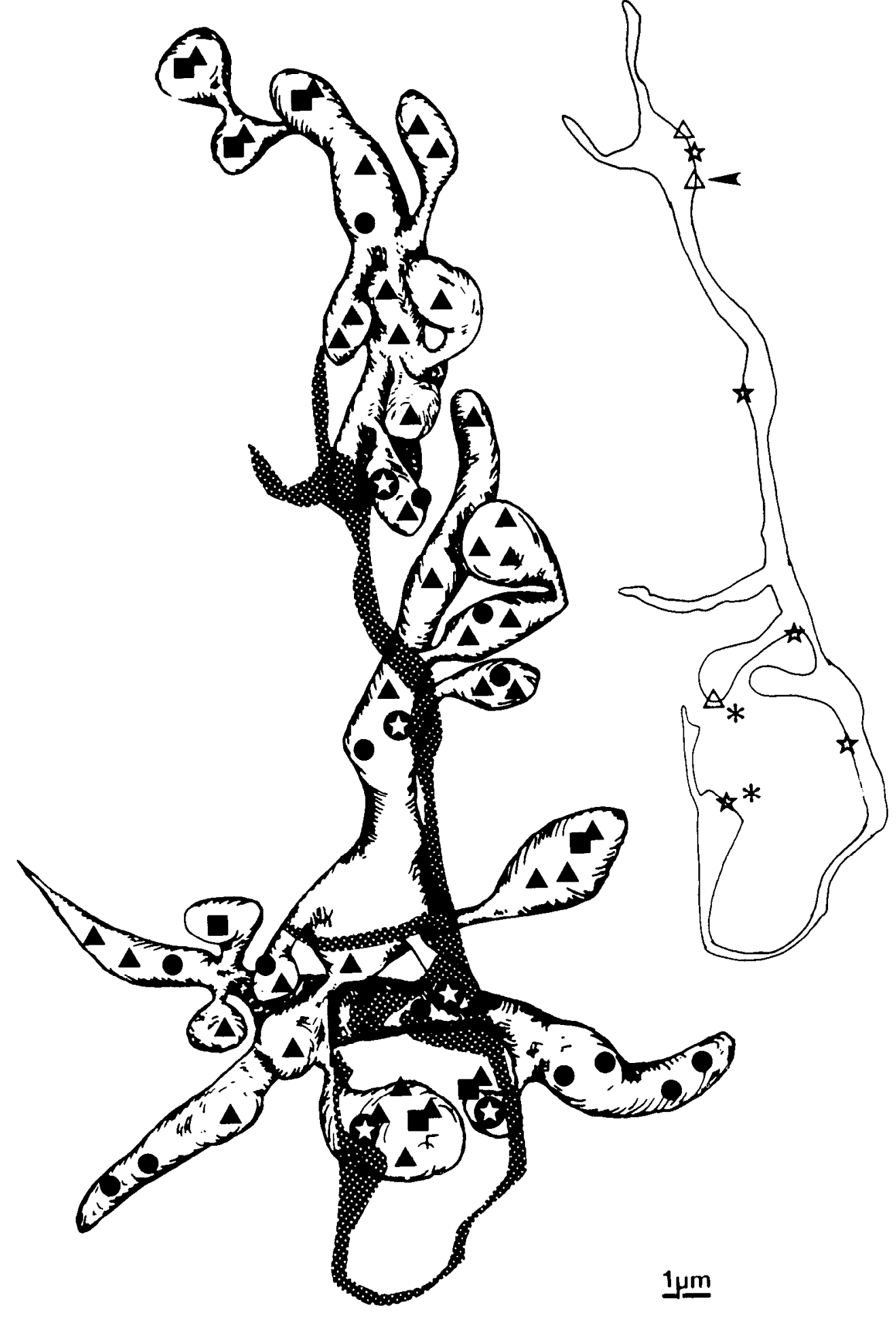

On average, parabrachial terminals have narrower synaptic clefts than do RSD terminals $(p<0.001)$, RLP terminals $(p<$ $0.001), \mathrm{F} 2$ terminals $(p<0.01), \mathrm{F} 1$ terminals $(p<0.05)$, and labeled cortical terminals $(p<0.001)$. Parabrachial terminals also display much less variation in synaptic cleft width than does each of the other terminal populations $(p<0.01$ on an $F$ test for each comparison), suggesting that the parabrachial axons labeled by our small PHA-L injection may be morc homogeneous than each of the other populations. Finally, as noted above for postsynaptic density, the differences in synaptic cleft parameters between labeled cortical and parabrachial terminals indicate that the labeling does not by itself dictate these parameters.

\section{Discussion}

Our data show that axons from the parabrachial region of the brain stem form conventional synaptic contacts onto neurons in the cat's lateral geniculatc nuclcus. Within the geniculate A-laminae, the parabrachial axons synapse onto dendritic shafts, 


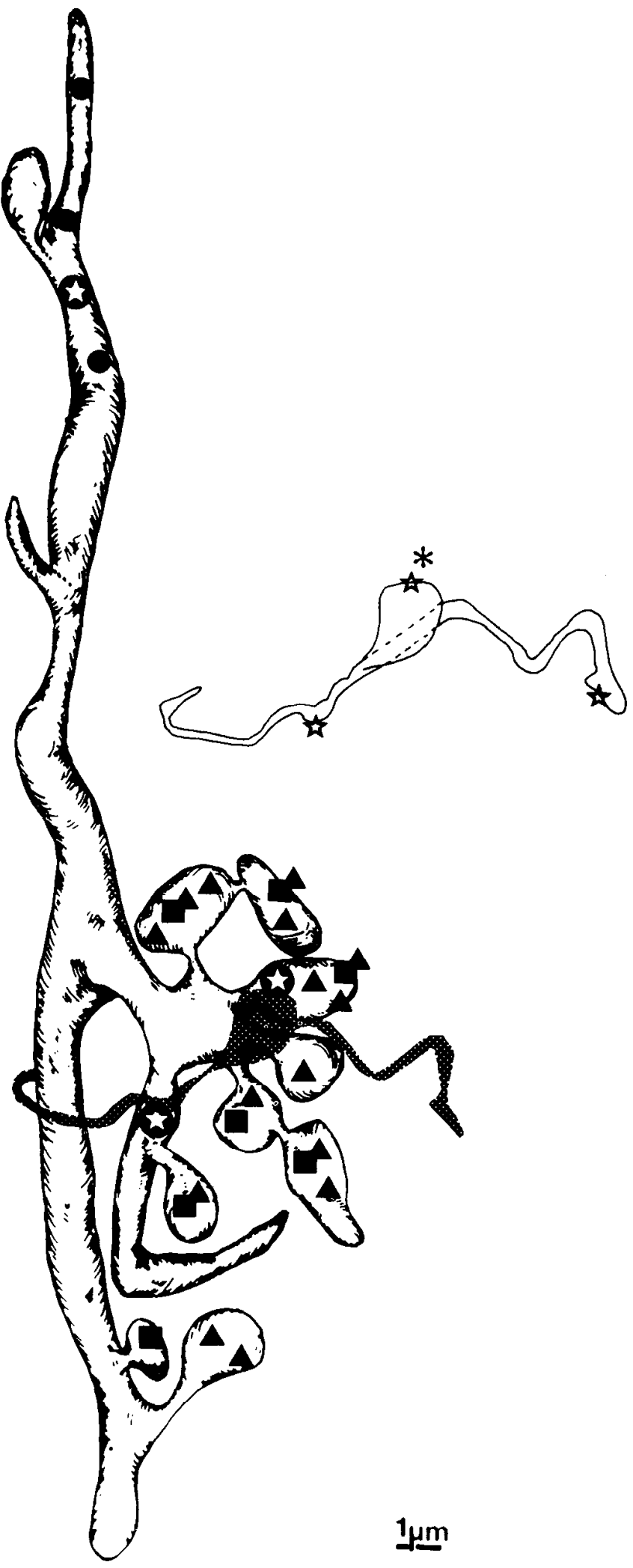

Figure 8. Serial reconstructions of a labeled parabrachial axon and a postsynaptic dendritic segment. Conventions are as in Figure 4. Note that this axon contacts no F2 terminals. The asterisk indicates the synapse shown in Figure $3 C$.

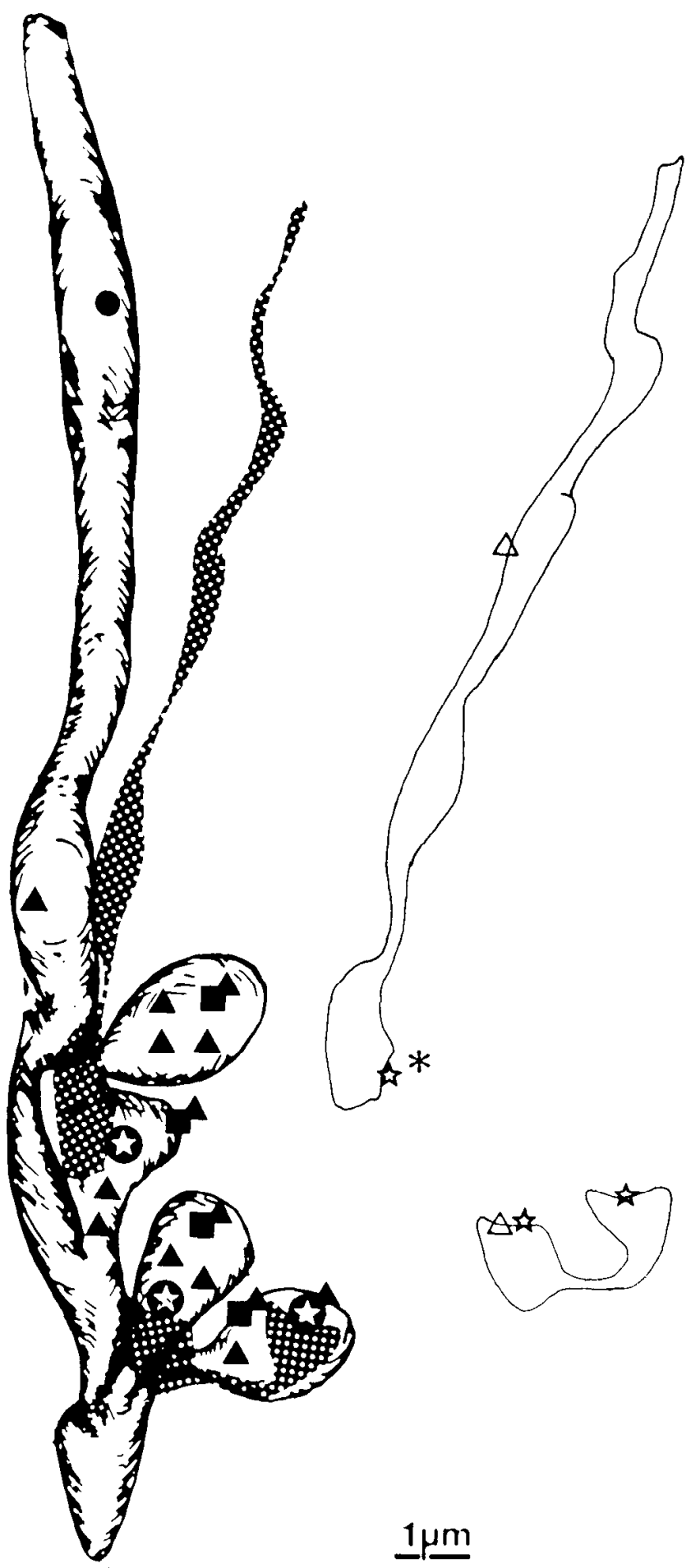

Figure 9. Serial reconstruction of portions of a labeled parabrachial axon and a postsynaptic dendritic segment. Conventions are as in Figure 4 . Although not completely connected, the 2 axonal segments probably belong to the same axon since this is the only labeled axon in this area of the neuropil. The parabrachial axon contacts both F2 terminals and dendritic appendages of a presumcd rclay $X$ cell. The asterisk indicates the synapse shown in Figure $3 B$. 


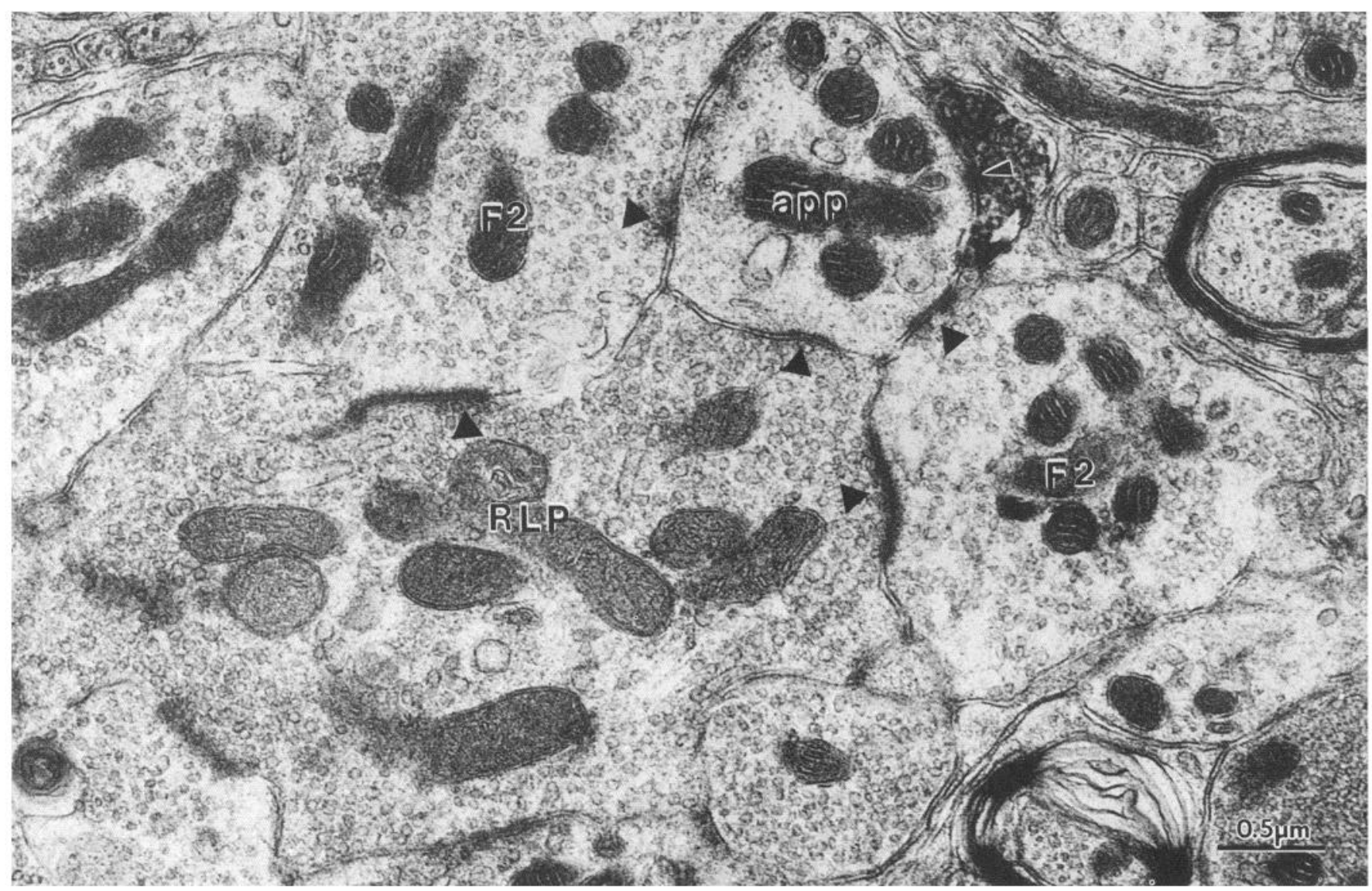

Figure 10. Electron micrograph of a synaptic glomerulus. The retinal terminal $(R L P)$ contacts $2 \mathrm{~F} 2$ terminals, and all 3 then contact the appendage (app) of a presumed relay X cell (solid triangles). A labeled parabrachial terminal (outlined triangle) also contacts the same appendage. At a different level through this glomerulus (not illustrated), the labeled axon also contacts an F2 terminal, which then contacts the same appendage. The parabrachial innervation is peripheral within the glomerulus relative to the retinal input.

dendritic appendages, and F2 terminals. Synapses from parabrachial terminals have remarkably narrow synaptic clefts compared with those of other terminal types. The parabrachial synapses formed onto dendritic shafts and F2 terminals have little postsynaptic density associated with the contact zone, while those formed onto dendritic appendages have more postsynaptic density. The possibility that these represent symmetrical and asymmetrical synapses is considered below. In any case, a single parabrachial axon can form both types of synapse. Finally, we have reconstructed portions of geniculate cell dendrites postsynaptic to the labeled axons. Many of these have clusters of complex dendritic appendages onto which retinal and F2 terminals form triadic synaptic contacts, often within glomeruli. Like the retinal input, the parabrachial axons frequently innervate the same glomeruli to form synaptic triadic relationships with the same F2 terminals and dendritic appendages.

\section{Nature of the brain-stem innervation}

More than $80 \%$ of the parabrachial neurons that can be retrogradely labeled after HRP injections into the lateral geniculate nucleus can be double-labeled with an antibody to choline acetyltransferase (De Lima and Singer, 1987; Smith et al., 1988). This suggests that most of the parabrachial cells innervating the lateral geniculate nucleus are cholinergic. It is thus likely that most of the axons that we have labeled with PHA-L are cho- linergic. The recent electron microscopic study of De Lima and colleagues (1985) is interesting in this regard. They analyzed synaptic terminals stained with antibodies against choline acetyltransferase in the geniculate A-laminae and reported that the stained terminals have synaptic relationships very similar to those we have described for parabrachial terminals in the present study. The cholinergic terminals often innervate glomerular zones, forming both symmetrical and asymmetrical synapses there, an individual cholinergic axons form synaptic contacts onto both relay cell appendages and F2 terminals in triadic relationships (De Lima et al., 1985).

Although it is tempting to view the present analysis and that of De Lima and colleagues (1985) as complementary studies of the same pathway, there are 2 alternative possibilities that bear scrutiny. First, it is possible that many of the terminals studied by De Lima and colleagues (1985) do not derive from the parabrachial region, since the locus coeruleus also projects a substantial number of cholinergic axons to the lateral geniculate nucleus (De Lima and Singer, 1987; Smith et al., 1988). Furthermore, other sources of cholinergic innervation, such as the basal forebrain and parabigeminal nucleus cannot be completely ruled out. Second, while our work identifies the parabrachial region as the source of labeled terminals, we cannot be certain of the transmitter employed by these terminals. To put this in a somewhat different context, it is possible that we and De Lima 


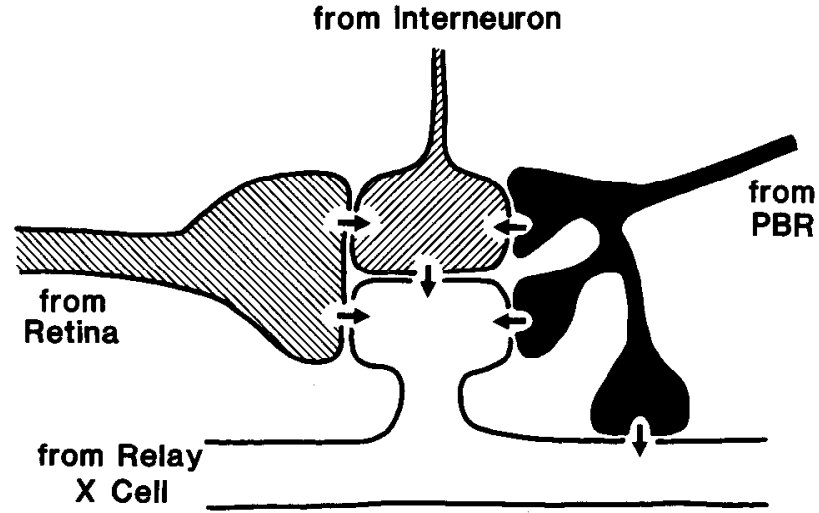

Figure 11. Schematic diagram showing the types of triadic input illustrated in Figure 7. A single parabrachial axon contacts a dendritic F2 terminal of an interneuron and both contact the appendage of a relay $X$ cell. The parabrachial axon also contacts the dendritic shaft of the same neuron. Likewise, a single retinal terminal contacts the same F2 terminal and dendritic appendage.

and colleagues (1985) have described the morphology of cholinergic innervation to the A-laminae from the parabrachial region, in which case a description of other pathways (e.g., the noradrenergic input from the brain stem and the input from the locus coeruleus) remains to be done. It is also possible that our 2 studies have identified different axonal populations that share a common morphological pattern of innervation within the lateral geniculate nucleus.

\section{Identification of parabrachial terminals}

As noted in Results, 4 major classes of synaptic terminal have been identified that account for $98 \%$ of the terminals found in the A-laminae of the cat's lateral geniculate nucleus (Guillery, $1969 \mathrm{a}, \mathrm{b}$ ). Of these, the RLP terminals are known to be retinal in origin and the F2 terminals are known to derive from dendritic appendages of interneurons. Thus, the RSD and F1 terminal populations represent virtually all of the other sources of extrinsic and intrinsic innervation to the A-laminae. We can consider how the parabrachial terminals would be identified.

In our material, the PHA-L reaction product is dark and the osmotic effects of the label cannot be determined, so we have not evaluated in detail the cytoplasmic density or vesicle shape in the parabrachial terminals. These important classification criteria were thus unavailable to us. We have instead analyzed the synaptic relationships of the labeled parabrachial terminals, the amount of postsynaptic density associated with their synaptic contact sites, and the widths of their synaptic clefts. The postsynaptic densities suggest that some parabrachial terminals might be identified as RSD terminals and others as F1 terminals (see Fig. 12). We have noted that unlabeled RSD and F1 terminals are often engaged in synaptic relationships that are similar to those we have described for the parabrachial terminals. However, our analysis of synaptic clefts (Fig. 13) suggests that the parabrachial terminals as a population are different from most RSD and Fl terminals, even though individual parabrachial terminals cannot be distinguished from the others on the basis of their synaptic clefts alonc. Furthermorc, cleft dimension is rarely used to identify synaptic terminals in the geniculate neuropil. We thus conclude that it is possible that some of the terminals identified as RSD or F1 in unlabeled material derive from the parabrachial region despite our evidence that the para-
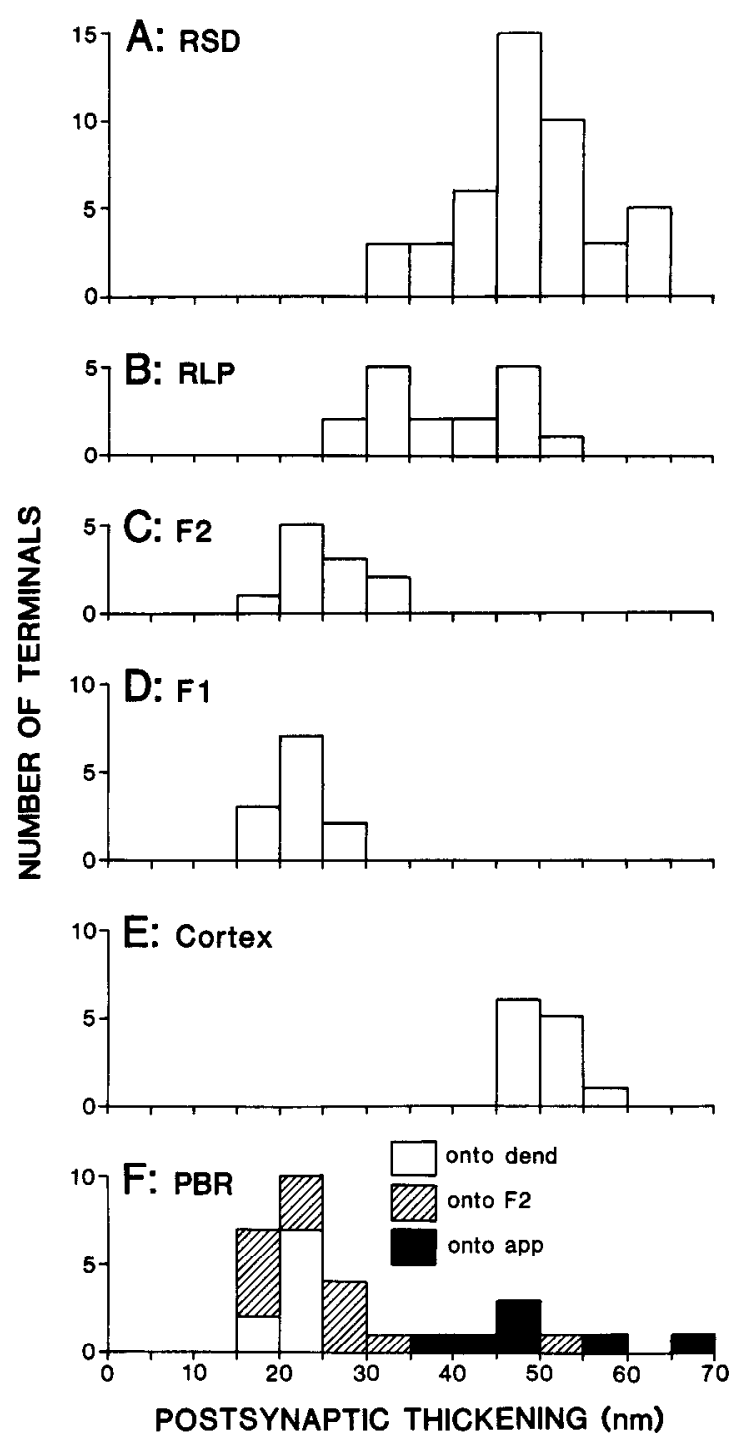

Figure 12. Histograms comparing the extent of postsynaptic density for different types of terminals, including RSD terminals $(A)$, RLP terminals $(B), \mathrm{F} 2$ terminals $(C), \mathrm{F} 1$ terminals $(D)$, terminals labeled with PHA-L from visual cortex $(E)$, and terminals labeled with PHA-L from the parabrachial region $(F)$. The terminals in $A-D$ are unlabeled. The bottom histogram also indicates whether each terminal forms a synapse onto a dendritic shaft (dend), an F2 terminal $(F 2)$, or a dendritic appendage (app).

brachial terminals as a population can be distinguished from RSD and F1 terminals. It is also possible that all of the parabrachial terminals might be included within the $2 \%$ of the terminal population that falls outside of the 4 main groups (Guillery, $1969 a, b)$.

It is interesting in this context that prior descriptions of RSD and F1 terminals within synaptic glomeruli are consistent with our descriptions of the parabrachial terminals. Relatively few RSD terminals are found in these glomeruli, and some of these terminals are known to be cortical in origin (Szentagothai, 1966; Guillery, 1969a, b; Jones and Powell, 1969). This does not rule out the possibility that some of the labeled parabrachial terminals exhibit RSD morphology, especially those contacting dendritic appendages (Weber and Kalil, 1987). Both RSD and parabrachial terminals are peripherally located within glomeruli, and, when present, the former most commonly contact den- 

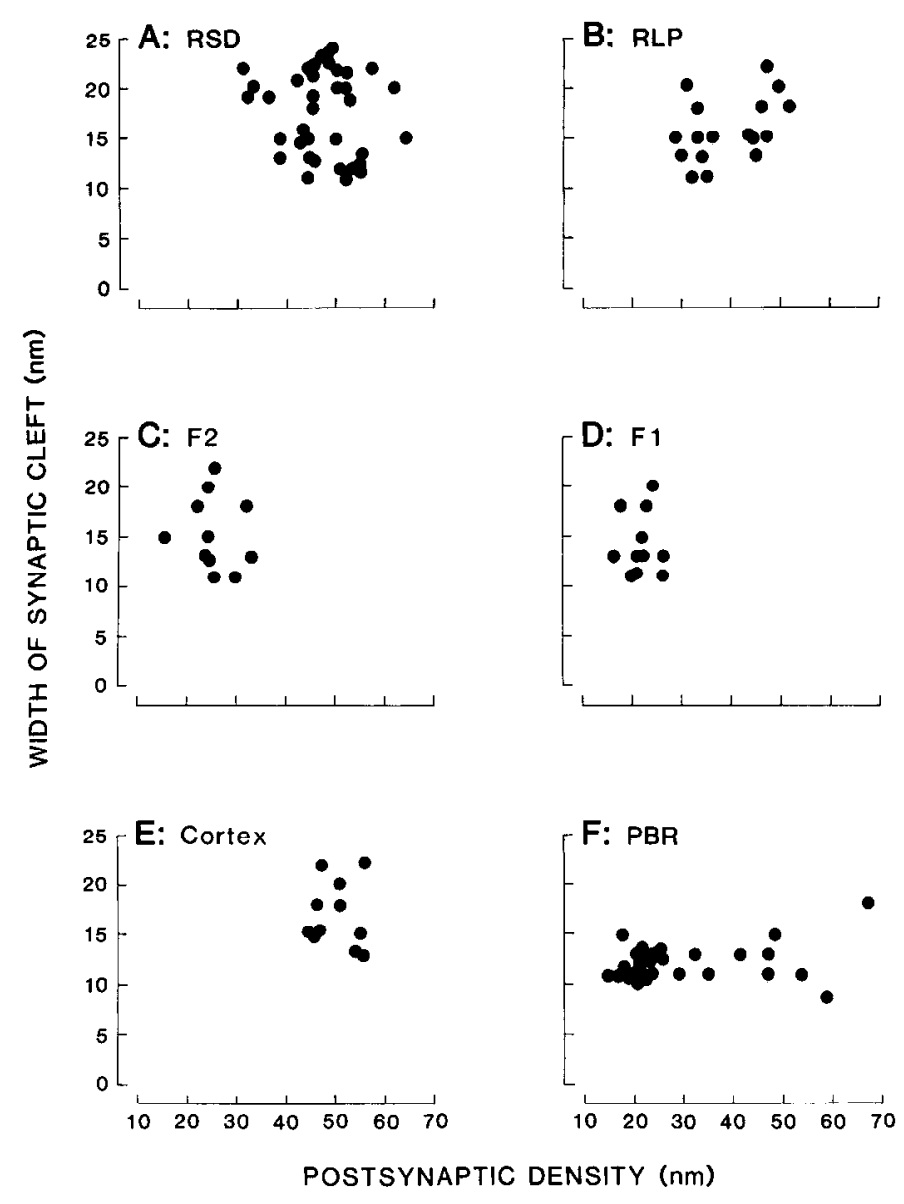

Figure 13. Scatter plots showing the relationship between the extent of postsynaptic density and the width of the synaptic cleft for various synaptic terminals, including RSD terminals $(A)$, RLP terminals $(B)$, $\mathrm{F} 2$ terminals $(C), \mathrm{F} 1$ terminals $(D)$, terminals labeled from visual cortex $(E)$, and terminals labeled from the parabrachial rcgion $(F)$.

dritic appendages (Guillery, 1969b). Based on these earlier reports of the paucity of RSD terminals in glomeruli, it may be that, if the parabrachial terminals there have RSD morphology, they are relatively rare. Likewise, F1 terminals are commonly seen peripherally within glomeruli and can contact $F 2$ terminals (Guillery, 1969a, b), which is precisely the pattern we have described for parabrachial terminals with symmetrical contacts. Although many F1 terminals may derive from other sources, e.g., from axons of interneurons or perigeniculate cells (see Cucchiaro et al., 1985; Montero, 1987), this does not preclude the possibility that some derive from parabrachial axons.

\section{Identification of postsynaptic geniculate cells}

Criteria developed mostly in our laboratory (Friedlander et al., 1981; Stanford et al., 1983; Wilson et al., 1984; Hamos et al., 1985, 1987; Humphrey and Weller, 1988) can be used with reasonable confidence to identify the cell type of origin for some of the unlabeled processes in the geniculate neuropil. These criteria are based on features of dendritic morphology and synaptic circuitry as determined with the light and electron microscopes. We can use these criteria to identify tentatively some of the neurons postsynaptic to the labeled parabrachial terminals.

The geniculocortical relay cells in the A-laminae can be divided into $X$ and $Y$ classes (for reviews, sec Stonc, 1983; Sherman, 1985). Relay Y cells tend to have fairly smooth dendrites and receive more than $90 \%$ of their retinal input onto dendritic shafts without triadic arangements; F2 terminals only rarely innervate $\mathrm{Y}$ cells, and glomeruli are generally not a feature of the $\mathrm{Y}$ pathway. In contrast, relay $\mathrm{X}$ cells tend to have clusters of dendritic appendages that are the primary site of retinal innervation. More than $90 \%$ of this retinal innervation typically involves triadic relationships with F2 terminals and often is embedded in complex synaptic glomeruli (Wilson et al., 1984; Hamos et al., 1985, 1987). As noted above, the F2 profiles are the specialized dendritic appendages of geniculate interneurons (Famiglietti and Peters, 1972; Hamos et al., 1985; Montero, 1986). The retinorecipient zone is thus characteristically different for $X$ and $Y$ cells, and this allows them to be distinguished ultrastructurally.

Labeled parabrachial terminals often contact F2 terminals, indicating that interneurons receive direct parabrachial input. We have also observed labeled terminals contacting structures in retinorecipient zones that fulfill all of the above-mentioned criteria for relay $\mathrm{X}$ cells. We have thus tentatively identified such structures as belonging to relay $X$ cells. This implies that at least some geniculate relay cells receive direct parabrachial inncrvation, and not all brain-stem influences on these cells are indirctly routed through interneurons and perigeniculate cells (cf. Ahlsen and Lo, 1982; Ahlsen et al., 1984). Interestingly, we have provisional evidence that the parabrachial innervation may select between the $X$ and $Y$ pathways. All of the dendritic segments that are postsynaptic to labeled parabrachial terminals and that have been adequately reconstructed to include their retinorecipient zones appear to be relay $\mathrm{X}$ cells. The parabrachial axons form synaptic contacts onto the appendages as well as onto the dendritic shafts of these segments. However, the parabrachial axons also contact numerous dendritic segments that have not yet been reconstructed to their retinorecipient zones. It is not possible to identify these cells without further reconstruction. These may represent other relay $X$ cells, relay $Y$ cells, or interneurons.

Brain-stem influences on the $Y$ pathway have been described with electrophysiological methods (Singer, 1973; Fukuda and Stone, 1976; Foote et al., 1977; Ahlsen et al., 1984), although we still lack anatomical evidence for this. We have not yet used the electron microscope to examine parabrachial innervation to magnocellular lamina $\mathrm{C}$ or the medial interlaminar nucleus, geniculate regions in which many $Y$ cells and few $X$ cells are found (see Stone, 1983; Sherman, 1985). In any case, our sample is small, and, as noted in the previous paper (Uhlrich et al., 1988), parabrachial axons are morphologically diverse with respect to terminal arbors and the sizes of terminal boutons. The labeled boutons reconstructed in the present paper are mostly at the small end of the size spectrum, consistent with a PHA-L injection limited to the caudal portion of the parabrachial region (see Uhlrich et al., 1988). This raises the possibility that our sample includes only a subset of parabrachial axons.

\section{Relationship between parabrachial and retinal synapses}

It is interesting that so many of the parabrachial synapses are located close to retinal synapses, at least for the postsynaptic neurons that we have identified as likely to be relay $\mathrm{X}$ cells. The parabrachial axons can influence these geniculate relay cells directly via contacts onto their dendritic appendages and shafts and indirectly via synapses onto the F2 terminals of interneurons. A parabrachial terminal frequently shares synaptic space with a retinal terminal on the same $\mathrm{F} 2$ terminal and dendritic 
appendage. Thus, the parabrachial innervation is often well sited to modulate retinal transmission through these geniculocortical relay cells. This is precisely the functional role attributed to this innervation (for review, see Sherman and Koch, 1986).

\section{Degrees of symmetry of synaptic contacts from parabrachial terminals}

As can be seen from Figure $12 F$, parabrachial terminals form synapses that have a wide range of postsynaptic densities. This range encompasses that of synapses from RSD and RLP terminals, on the one hand, and of those from F2 and F1 synapses, on the other. Synapses from RSD and RLP terminals are asymmetrical, while those from $\mathrm{F} 1$ and $\mathrm{F} 2$ terminals are symmetrical. It is thus tempting to conclude that parabrachial synapses can be either symmetrical or asymmetrical, and we shall refer to them as such. There is a proviso to this terminology. In the cerebral cortcx, several parameters are reported to correlate with the symmetry of synaptic contacts: A terminal forming a symmetrical synapse tends to have flattened or pleomorphic vesicles and a narrow synaptic cleft; one forming an asymmetrical synapse tends to have spherical vesicles and a wide synaptic cleft (Gray, 1959; Colonnicr, 1968; for discussion, see Peters et al., 1976). Recently, Weber and Kalil (1987) reported that autoradiographically labeled brain-stem terminals in the geniculate neuropil of the cat have round vesicles. However, as noted above, we cannot analyzc vesicle shape in the labeled terminals, and the geniculate neuropil generally lacks the relationship found in cortex between synaptic symmetry and the width of the synaptic cleft (see Fig. 13, $A-D$; see also Gray and Guillery, 1966; Rapisardi and Lipsenthal, 1984). For these reasons, we have relied on the extent of the postsynaptic density for our classification of parabrachial synapses.

We observed single, labeled axons that produce both asymmetrical and symmetrical synaptic contacts. This runs counter to the conventional wisdom that asymmetrical synapses denote cxcitatory action while symmetrical synapses are inhibitory and that each axon should form only one type of synapse. We found that the symmetry of the synapse depends on the specific postsynaptic element innervated. Synaptic contacts from parabrachial axons onto dendritic appendages are the most asymmetrical, while those onto both F2 terminals and dendritic shafts are the most symmetrical.

The symmetry of these synaptic contacts thus seems not to depend solcly on the axon. It is intereting that the putative neurotransmitters for these axons, ACh and noradrenaline, can have different postsynaptic actions depending on the postsynaptic receptor (see review in Sherman and Koch, 1986). Perhaps the variation in postsynaptic density seen for parabrachial synapses may, in some as yet unspecified manner, reflect different receptor proteins in the postsynaptic membranes. For instance, if these parabrachial axons use $A C h$ as a neurotransmitter, then different postsynaptic densities might reflect the presence of different receptors. Two different types of muscarinic receptor and one nicotinic type, all of which respond to $\mathrm{ACh}$ but in quite different ways, have been identified in the A-laminae of the cat's lateral geniculate nucleus (McCormick and Prince, 1987). A related suggestion stems from studies in the rat showing that more than $20 \%$ of the parabrachial neurons that give rise to an ascending projection contain both $\mathrm{ACh}$ and substance P (Vincent et al., 1983, 1986). Perhaps a single axon contains different mixes of neurotransmitters, and different postsynaptic receptors determine which neurotransmitter will act. In any case, the mor- phology we have described suggests that the synaptic action of a single axon may vary with the postsynaptic partner, an it may even differ at different sites on the same neuron.

\section{References}

Ahlsen. G., and F.-S. Lo (1982) Projection of brainstem neurones to the perigeniculate nucleus and the lateral geniculate nucleus in the cat. Brain Res. 238: 433-438.

Ahlsen, G., S. Lindstrom, and F.-S. Lo (1984) Inhibition from the brainstem of inhibitory interneurones of the cat's dorsal lateral geniculate nucleus. J. Physiol. (Lond.) 347: 593-609.

Burke, W., and A. M. Cole (1978) Extraretinal influences on the lateral geniculate nucleus. Rev. Physiol. Biochem. Pharmacol. 80: 105-166.

Colonnier, M. (1968) Synaptic patterns on different cell types in the different laminae of the cat visual cortex. An electron microscope study. Brain Res. 9: 268-287.

Cucchiaro, J. B., D. J. Uhlrich, J. E. Hamos, and S. M. Sherman (1985) Perigeniculate input to the cat's lateral geniculate nucleus: A light and electron microscopic study of single, HRP filled cells. Soc. Neurosci. Abstr. 11: 231 .

Cucchiaro, J. B., D. J. Uhlrich, and S. M. Sherman (1986) Brainstem innervation to the cat's lateral geniculate nucleus: An EM-immunohistochemical study using the tracer Phaseolus vulgaris leucoagglutinin (PHA-L). Soc. Neurosci. Abstr. 12: 1037.

De Lima, A. D., and W. Singer (1987) The brainstem projection to the lateral geniculate nucleus in the cat: Identification of cholinergic and monoaminergic clements. J. Comp. Neurol. 259: 92-121.

De Lima, A. D., V. M. Montero, and W. Singer (1985) The cholinergic innervation of the visual thalamus: An EM immunocytochemical study. Exp. Brain Res. 59: 206-212.

Eldred, W. D., C. Zucker, H. J. Karten, and S. Yazulla (1983) Comparison of fixation and penetration enhancement techniques for use in ultrastructural immunohistochemistry. J. Histochem. Cytochem. 31: $285-292$.

Eyscl, U. T., H. C. Pape, and R. Van Schayck (1986) Excitatory and differential disinhibitory actions of acetylcholine in the lateral geniculate nucleus of the cat. J. Physiol. (Lond.) 370: 233-254.

Famiglietti, E. V.. and A. Peters (1972) The synaptic glomerulus and the intrinsic neuron in the dorsal lateral geniculate nucleus of the cat. J. Comp Neurol. 144: 285-334.

Foote, W. E., J. P. Mordes, C. L. Colby, and T. A. Harrison (1977) Differential effect of midbrain stimulation on $\mathrm{X}$-sustained and $\mathrm{Y}$-transient neurons in the lateral geniculate nucleus of the cat. Brain Res. 127: $153-158$

Friedlander, M. J.. C.-S. Lin, L. R. Stanford. and S. M. Sherman (1981) Morphology of functionally identified neurons in the lateral geniculate nucleus of the cat. J. Neurophysiol. 46:80-129.

Fukuda, Y., and J. Stone (1976) Evidence of differential inhibitory influences on $\mathrm{X}$ - and $\mathrm{Y}$-type relay cells in the cat's lateral geniculate nucleus. Brain Res. 113: 188-196.

Gerfen. C., and P. E. Sawchenko (1984) An anterograde neuroanatomical tracing method that shows the detailed morphology of neurons, their axons and terminals: Immunohistochemical localization of an axonally transported plant lectin, Phaseolus vulgaris leucogglutinin (pha-1). Brain Res. 290: 219-238.

Gray, E. G. (1959) Axo-somatic and axo-dendritic synapses of the cerebral cortex: An electron microscope study. J. Anat. 93: 420-433.

Gray, E. G., and R. W. Guillery (1966) Synaptic morphology in the normal and degenerating nervous system. Int. Rev. Cytol. 19: 111182.

Guillery, R. W. (1969a) The organization of synaptic interconnections in the laminae of the dorsal lateral geniculate nucleus of the cat. $Z$. Zellforsch. 96: 1-38.

Guillery, R. W. (1969b) A quantitative study of synaptic interconnections in the dorsal lateral geniculate nucleus of the cat. Z. Zellforsch. 96: 39-48.

Hamos, J. E., S. C. Van Horn, D. Račkowski, D. J. Uhlrich, and S. M. Sherman (1985) Synaptic connectivity of a local circuit neurone in the lateral geniculate nucleus of the cat. Nature 317:618-621.

Hamos, J. E., S. C. Van Horn, D. Račkowski, and S. M. Sherman (1987) Synaptic circuits involving an individual retinogeniculate axon in the cat. J. Comp. Neurol. 259: 165-192.

Hughes, H. C., and W. H. Mullikin (1984) Brainstem afferents to the lateral geniculate nucleus of the cat. Exp. Brain Res. 54: 253-258. 
Humphrey, A. L., and R. E. Weller (1988) Structural correlates of functionally distinct $X$-cells in the lateral geniculate nucleus of the cat. J. Comp. Neurol. 268: 448-468.

Jones, E. G., and T. P. S. Powell (1969) An electron microscopic study of the mode of termination of cortico-thalamic fibres within the sensory relay nuclei of the thalamus. Proc. R. Soc. London [Biol.] 172: 173-185.

Leger, L., K. Sakai, D. Salvert, M. Touret, and M. Jouvet (1975) Delineation of the dorsal lateral geniculate afferents from the cat brainstem visualized by the horseradish peroxidase technique. Brain Res. 93: 490-496.

McCormick, D. A., and D. A. Prince (1987) Actions of acetylcholine in the guinea pig and cat medial and lateral geniculate nuclei in vitro. J. Physiol. (Lond.) 392: 147-165.

Montero, V. M. (1986) Localization of gamma-aminobutyric acid (GABA) in type 3 cells and demonstration of their source to F2 terminals in the cat lateral geniculate nucleus: A Golgi-electron microscopic GABA-immunocytochemical study. J. Comp. Neurol. 254: $228-245$.

Montero, V. M. (1987) Ultrastructural identification of synaptic terminals from the axon of type 3 interneurons in the cat lateral geniculate nucleus. J. Comp. Neurol. 264: 268-283.

Peters, A., S. L. Palay, and H. deF. Webster (1976) The Fine Structure of the Nervous System: The Neurons and Supporting Cells, Saunders, Philadelphia.

Rapisardi, S. C., and L. Lipsenthal (1984) Asymmetric and symmetric synaptic junctions in the dorsal lateral geniculate nucleus of cat and monkey. J. Comp. Neurol. 224: 415-424.

Robson, J. A. (1983) The morphology of corticofugal axons to the dorsal lateral geniculate nucleus in the cat. J. Comp. Neurol. 216:89103.

Robson, J. A., and C. A. Mason (1979) The synaptic organization of terminals traced from individual labeled retino-geniculate axons in the cat. Neuroscience 4: 99-111.

Sherman, S. M. (1985) Functional organization of the $\mathrm{W}-, \mathrm{X}$ - and $\mathrm{Y}$-cell pathways in the cat: A review and hypothesis. In Progress in Psychobiology and Physiological Psychology, Vol. 2, pp. 233-314, Academic, New York.

Sherman, S. M., and C. Koch (1986) The control of retinogeniculate transmission in the mammalian lateral geniculate nucleus. Exp. Brain Res. 63: $1-20$.

Sillito, A. M., J. A. Kemp, and N. Berardi (1983) The cholinergic influence on the function of the cat dorsal lateral geniculate nucleus (dLGN). Brain Res. 280: 299-307.

Singer, W. (1973) Brain stem stimulation and the hypothesis of presynaptic inhibition in cat lateral geniculate nucleus. Brain Res. 61: $55-68$.

Singer, W. (1977) Control of thalamic transmission by corticofugal and ascending reticular pathways in the visual system. Physiol. Rev. 57: $386-420$.

Smith, Y., D. Pare, M. Deschenes, A. Parent, and M. Steriade (1988) Cholinergic and non-cholinergic projections from the upper brainstem core to the visual thalamus in the cat. Exp. Brain Res. 70: 166-180.

Stanford, L. R., M. J. Friedlander, and S. M. Sherman (1983) Morphological and physiological properties of geniculate W-cells of the cat: A comparison with X-and Y-cells. J. Neurophysiol. 50: 582608.

Stone, J. (1983) Parallel Processing in the Visual System, Plenum, New York.

Szentagothai, J., J. Hamori, and T. Tombol (1966) Degeneration and electron microscope analysis of the synaptic glomeruli in the lateral geniculate body. Exp. Brain Res. 2: 283-301.

Uhlrich, D. J., J. B. Cucchiaro, and S. M. Sherman (1988) The projection of individual axons from the parabrachial region of the brain stem to the dorsal lateral geniculate nucleus in the cat. J. Neurosci. 8: $4565-4575$.

Vincent, S. R., K. Satoh, D. M. Armstrong, and H. C. Fibiger (1983) Substance $\mathrm{P}$ in the ascending cholinergic system. Nature 306: 688691.

Vincent, S. R., K. Satoh, D. M. Armstrong, P. Panula, W. Wale, and H. A. Fibiger (1986) Neuropeptides and NADPH-diaphorase activity in the ascending cholinergic reticular system of the rat. Neuroscience 17: 167-182.

Weber, A. J., and R. E. Kalil (1987) Development of corticogeniculate synapses in the cat. J. Comp. Neurol. 264: 171-192.

Wilson, J. R., M. J. Friedlander, and S. M. Sherman (1984) Fine structural morphology of identified X-and Y-cells in the cat's lateral geniculate nucleus. Proc. R. Soc. London [Biol.] 221: 411-436. 